\title{
TAXA DE DESENVOLVIMENTO DE FORÇA E ATIVAÇÃO NEURAL EM MULHERES PÓS-MENOPAUSADAS
}

\author{
RATE OF FORCEDEVELOPMENT AND NEURAL DRIVE IN POSTMENOPAUSAL WOMEN
}

TASA DE DESARROLLO DE LA FUERZA Y ACTIVACIÓN NEURONAL EN MUJERES POSMENOPÁUSICAS

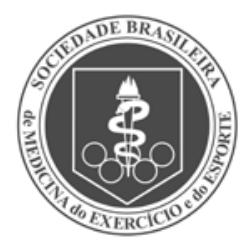

Artigo Original

Original ARTICLE Artículo Original
Piettra Moura Galvão Pereira 1,2 (Educadora Física)

André Luiz Ferreira de Araújo ${ }^{1}$ (Graduando em Educação Física) Elys Reginna Lopes de Oliveira' (Graduanda em Educação Física)

Maria da Glória David Silva Costa (Graduanda em Educação Física)

Amandio Aristides Rihan Geraldes' (Educador Físico)

Maria do Socorro Cirilo-Sousa ${ }^{2}$ (Educadora Física)

1. Universidade Federal de Alagoas, Centro de Educação, Laboratório de Aptidão Física, Desempenho e Saúde, Maceió, AL, Brasil.

2. Universidade Federal da Paraíba, Centro de Ciências da Saúde,

Núcleo de Pesquisa em Ciências do Movimento Humano, Laboratório de Cineantropometria e Desempenho Humano, João Pessoa, PB, Brasil.

\section{Correspondência:}

Cidade Universitária, Centro de Ciências da Saúde, Núcleo de Pesquisas do Movimento Humano, Campus I, s/n - Castelo Branco, João Pessoa - PB, Brasil. 58051-900. piettra_galvao@hotmail.com

\section{RESUMO}

Introdução: O envelhecimento associa-se à redução da velocidade de contração e ativação das fibras musculares, influenciando a aptidão física e o desempenho funcional. Objetivo: Verificar o comportamento da taxa de desenvolvimento de força (TDF) e ativação neural em mulheres pós-menopausadas. Métodos: Vinte e quatro mulheres pós-menopausadas ( $63,2 \pm 5,6$ anos; $154,5 \pm 7,3 \mathrm{~cm}$ e 64,7 \pm 7,6 kg), funcionalmente independentes e fisicamente ativas, foram submetidas simultaneamente, à mensuração da força isométrica máxima e dos sinais eletromiográficos (EMG) dos seguintes músculos: vasto lateral (VL), reto femoral (RF) e vasto medial (VM), durante uma extensão unilateral do joelho do membro dominante. Tais resultados foram utilizados para calcular a TDF, o impulso contrátil, em intervalos de 20 ms nos primeiros $200 \mathrm{~ms}$ da contração muscular pelo uso da curva força/tempo, a taxa de ativação EMG (TAE) e a amplitude média EMG, em intervalos de 40 a 80 ms do início da integração EMG pelo uso da curva EMG/tempo. Resultados: Os valores da força variaram entre 29,19 a 86,04 N.m; a TDF variou de 1459,42 N.m.s-1 para 430,21 N.m.s-1 em 20 ms e 200 ms respectivamente, apontando valores decrescentes com o tempo; para o impulso foram observados valores entre 0,65 a 11,07 N.m.s; na mesma direção da TDF, a TAE apresentou valores decrescentes para o VL

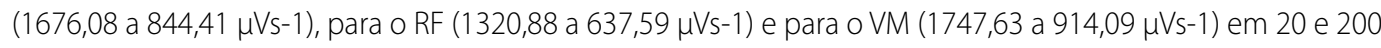
ms, enquanto a amplitude EMG média teve valores de 33,77 a 50,32 $\mu \mathrm{V}$ para o VL, de 24,93 a 38,07 $\mu \mathrm{V}$ para o RF e de 37,07 a 54,78 $\mu \mathrm{V}$ para o VM em 40 ms e 80 ms, respectivamente. Conclusão: Em mulheres pós-menopausadas, a velocidade de aumento, a manutenção da força e a ativação EMG não são suficientes para manter TDF e TAE crescentes, demonstrando um possível risco de incapacidades funcionais e ocorrência de quedas.

Descritores: envelhecimento; força muscular; eletromiografia.

\section{ABSTRACT}

Introduction: Aging is associated with a reduction in the speed of contraction and activation of muscle fibers, influencing physical fitness and functional performance. Objective: To verify the rate of force development (RFD) and neural activation in postmenopausal women. Methods: Twenty-four postmenopausal women (63.2 \pm 5.6 years; $154.5 \pm 7.3 \mathrm{~cm}$ and $64.7 \pm 7.6 \mathrm{~kg}$ ) functionally independent and physically active were submitted simultaneously to the measurement of maximal isometric force and electromyographic signals (EMG) of the following muscles: vastus lateralis (VL), rectus femoris (RF), and vastus medialis (VM) for a unilateral extension of the knee of the dominant limb. These results were used to calculate the RFD, the contractile impulse in 20-ms intervals during the first 200ms of the muscle contraction by the force/time curve, the EMG activation rate (EAR), and the mean EMG amplitude, at intervals of 40 to $80 \mathrm{~ms}$ from the start of the EMG integration by using the EMG/time curve. Results: The force values ranged from 29.19 to $86.04 \mathrm{Nm}$; the RFD varied from 1459.42 to $430.21 \mathrm{~N} . \mathrm{m} . \mathrm{s}-1$ in $20 \mathrm{~ms}$ and $200 \mathrm{~ms}$, respectively, indicating decreasing values with time; for the impulse values were observed between 0.65 and $11.07 \mathrm{Nms}$; in the same direction of RFD, the EAR showed decreasing values for the VL (1676.08 to 844.41 $\mu \mathrm{Vs}-1)$, for the RF (1320.88 to

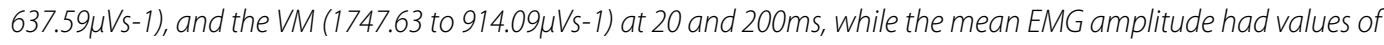
33.77 to $50.32 \mu \mathrm{V}$ for the $\mathrm{VL}$, 24.93 to $38.07 \mu \mathrm{V}$ for $R F$, and 37.07 to $54.78 \mu \mathrm{V}$ for the $V M$ in $40 \mathrm{~ms}$ and $80 \mathrm{~ms}$, respectively. Conclusion: In postmenopausal women, the increase rate, the maintenance of force, and the EMG activation are not sufficient to maintain the increase of RFD and EAR, demonstrating a potential risk of functional disabilities and falls.

Keywords: aging; muscle strength; electromyography.

\section{RESUMEN}

Introducción: El envejecimiento se asocia con la reducción de la velocidad de contracción y la activación de las fibras musculares, que influyen la aptitud física y el rendimiento funcional. Objetivo: Comprobar la tasa de desarrollo de la fuerza (TDF) y la activación de las fibras musculares en mujeres posmenopáusicas. Métodos: Veinticuatro mujeres posmenopáusicas (63,2 $\pm 5,6$ años; $154,5 \pm 7,3 \mathrm{~cm}$ y $64,7 \pm 7,6 \mathrm{~kg})$, con independencia funcional y físicamente activas, fueron sometidas simultáneamente a la medición de la fuerza isométrica máxima y de las señales electromiográficas de los siguientes músculos: vasto lateral (VL), recto femoral (RF) y vasto medial (VM) para una extensión unilateral de la rodilla de la extremidad dominante. Estos resultados se utilizaron para calcular la TDF y el impulso contráctil en intervalos de 20 ms durante los primeros 200 ms de la contracción del 
músculo mediante la curva fuerza/tiempo, la tasa de activación EMG (TAE) y la amplitud EMG media, a intervalos de 40 a 80 ms del inicio de la integración EMG, mediante el uso de la curva EMG/tiempo. Resultados: Los valores de fuerza variaron desde 29,19 hasta 86,04Nm; la TDF varió de 1.459,42 N.m.s-1 hasta 430,21 N.m.s-1 en 20 ms y $200 \mathrm{~ms}$, respectivamente, lo que indica valores decrecientes con el tiempo; para el impulso, se observaron valores entre 0,65 y 11,07 N.m.s; en la misma dirección de la TDF, la TAE mostró valores decrecientes para VL (1676,08 a $844,41 \mu \mathrm{Vs}-1)$, para RF (1320,88 a 637,59 $\mu \mathrm{Vs}-1)$ y para VM (1747,63-914,09 $\mu \mathrm{Vs}-1)$ a 20 y $200 \mathrm{~ms}$, mientras que el promedio de la amplitud EMG tuvo valores de 33,77 a 50,32 $\mu \mathrm{V}$ para VL, de 24,93 a 38,07 $\mu \mathrm{V}$ para RFy 37,07 a $54,78 \mu \mathrm{V}$ para VM en $40 \mathrm{~ms}$ y $80 \mathrm{~ms}$, respectivamente. Conclusión: En las mujeres posmenopáusicas, la velocidad del aumento, el mantenimiento de la fuerza y la activación EMG no son suficientes para mantener la TDF y la TAE crecientes, lo que demuestra un posible riesgo de discapacidad funcional y caídas.

Descriptores: envejecimiento; fuerza muscular; electromiografía.

\section{INTRODUÇÃO}

Concomitantemente com o processo de envelhecimento, ocorrem importantes alterações neuromusculares que podem influenciar a saúde e a qualidade de vida dos idosos ${ }^{1,2}$ ao reduzir a independência funcional para a realização das tarefas diárias ${ }^{3}$, aumentar os riscos de quedas, fraturas, imobilizações, internação, isolamento social, depressão e mortalidade por todas as causas ${ }^{4}$.

Dentre as possíveis alterações neuromusculares decorrentes do envelhecimento, destaca-se a perda gradual dos neurônios motores espinhais ${ }^{5,6}$, responsável pela redução no tamanho e número de fibras musculares ${ }^{6}$, podendo resultar em uma diminuição significante da massa e força muscular, condições estas, denominadas de sarcopenia e/ou dinapenia?.

Devido à sarcopenia, as fibras musculares de contração rápida, ou tipo II, são as mais prejudicadas pela denervação dos neurônios motores (NM), quando comparadas com as fibras de contração lenta ou tipo ${ }^{8}$. Em termos de desempenho funcional, tal fato cresce em importância, haja vista que essas fibras têm papel de destaque em tarefas motoras que necessitem de movimentos velozes e/ou de força, como por exemplo, em ações relacionadas com a recuperação do equilíbrio ${ }^{9}$ ou durante a realização de tarefas simples do dia a dia, como caminhar ou subir escadas ${ }^{6}$.

A velocidade inicial do movimento é determinada pela taxa de desenvolvimento de força (TDF), obtida pela curva força/tempo, variável dependente de uma maior estimulação das fibras do tipo $\|^{8}$. Em relação ao componente neural, algumas das alterações ocorridas relacionadas à idade podem ser observadas por meio da taxa de ativação eletromiográfica (TAE) ${ }^{6}$ ao mensurar-se a amplitude e duração dos potenciais de ação da unidade motora pelo uso da eletromiografia de superfície (EMG) ${ }^{10}$.

As alterações neuromusculares relacionadas ao envelhecimento são dependentes do sexo e apesar de os homens apresentarem maior velocidade de redução da força e da potência muscular, as mulheres podem ser mais vulneráveis, por apresentarem menor volume de massa muscular inicial, por apresentarem maior expectativa de vida e elevadas taxas de limitações funcionais ${ }^{11}$. Portanto, o conhecimento sobre o comportamento da TDF e da TAE, em muIheres pós-menopausadas, além de ampliar o conhecimento sobre a temática, pode contribuir para desenvolvimento das estratégias para a manutenção ou desaceleração das perdas musculares relacionadas com os efeitos deletérios do envelhecimento sobre a função neuromuscular e sobre o desempenho funcional.

Diante do exposto, o presente estudo teve como objetivo, verificar o comportamento da TDF e ativação neural em mulheres pós-menopausadas.

\section{MÉTODOS}

Vinte e quatro mulheres pós-menopausadas $(63,2 \pm 5,6$ anos; $154,5 \pm 7,3 \mathrm{~cm}$ e $64,7 \pm 7,6 \mathrm{~kg}$ ), funcionalmente independentes e fisicamente ativas no lazer, participaram da amostra deste estudo descritivo.

Para participar do estudo, as voluntárias não deveriam ser praticantes de treinamento de força (pelo menos nos últimos seis meses); serem normotensas ou terem a pressão arterial controlada, não relatarem lesões musculares ou articulares nos membros inferiores e serem capazes de entender os objetivos do estudo, bem como, as explicações relacionadas à realização do mesmo.

A pesquisa foi aprovada pelo Comitê de Ética em Pesquisa da Universidade Federal de Alagoas sob o número de protocolo 817.007, de acordo com a Resolução 466/12 do Conselho Nacional de Saúde. Todas as voluntárias assinaram o Termo de Consentimento Livre e Esclarecido.

A mensuração da força isométrica máxima e o sinal EMG foram avaliados simultaneamente. Durante a contração isométrica voluntária máxima (CIVM) foram a realizadas três tentativas do movimento de extensão do joelho do membro dominante, em um ângulo articular de $60^{\circ}$ (sendo o " 0 " correspondente a uma extensão completa) mensurado com auxílio de um goniômetro eletrônico (EMG System do Brasil, SP Ltda). A contração deveria ser mantida por cinco segundos e, em termos eletromiográficos, considerou-se o início da contração muscular, quando o torque (T) superou os sete e meio N.m. Para todas as variáveis, foi analisada a tentativa de teste que apresentou maior valor da CIVM.

Os testes foram realizados em uma cadeira de Bonnet adaptada, para evitar movimentos indesejáveis durante a realização do teste, o quadril foi fixado à cadeira com auxílio de cintas de lona. Nessa posição, o tornozelo era fixado à uma célula de carga (EMG System do Brasil, SP Ltda) com auxílio de uma braçadeira (localizada acima dos maléolos), ligada a uma corrente presa na extremidade fixa da cadeira.

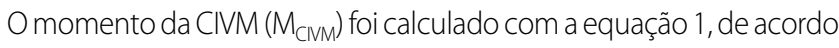
com os procedimentos propostos por Aagaard et al ${ }^{12}$.

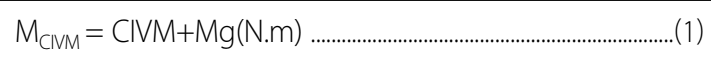

A correção pelo momento da gravidade $(\mathrm{Mg})$ foi realizada conforme estudos semelhantes ${ }^{12-14}$, com auxílio da equação 2.

$$
\mathrm{Mg}=\mathrm{m} . \mathrm{r} \cdot \operatorname{sen} \theta
$$

A massa da perna e do pé descrita por " $m$ "foi estimada pela equação 3 , que utiliza valores constantes publicados anteriormente ${ }^{15}$.

$m=0,0061^{*}$ massa corporal+massa da corrente......(3) 
A distância do eixo para o centro de massa foi denominada por "r", descrito na equação 4. E $\theta$ é o ângulo formado entre o local da braçadeira na perna e o plano vertical ${ }^{15}$.

$r=0,606^{*}$ comprimento da perna

Imediatamente antes do início do teste os sujeitos realizaram uma sessão de familiarização e após três minutos de intervalo realizaram o teste propriamente dito. Todos os sujeitos foram instruídos a realizar a contração o mais rápido e vigorosamente possível ${ }^{16}$, recebendo durante a contração o encorajamento verbal dos avaliadores.

O cálculo da TDF foi obtido através dos valores médios da curva momento/tempo ( $\Delta$ momento/ $\Delta$ tempo) durante os primeiros $200 \mathrm{~ms}$ de contração, em intervalos de tempo de $20 \mathrm{~ms}$, sendo o impulso contrátil definido como a área sob a curva momento/tempo de $20 \mathrm{~ms}$ até $200 \mathrm{~ms}$ da contração muscular ${ }^{13}$.

Antes da realização do teste, os locais dos eletrodos foram cuidadosamente demarcados, realizou-se a tricotomia, abrasão e a limpeza da pele com álcool nos locais onde os eletrodos seriam fixados.

Os sinais EMG foram mensurados com eletrodos de superfície (Ag/ AgCl-Meditrace 3M, SP, Brasil), simples diferencial, pré-amplificados, com uma distância inter-eletrodos de $20 \mathrm{~mm}$, posicionados nos ventres e longitudinalmente à direção das fibras musculares do vasto lateral (VL), medial (VM) e reto femoral (RF), como recomendado pelo manual: Surface EMG for a Non-invasive Assessment of Muscles ${ }^{17}$. Os sinais foram amplificados pelo eletromiógrafo de superfície (EMG System do Brasil, SP Ltda).

Os dados foram tratados retirando-se o componente contínuo (off set) e filtrados com Butterworth de quarta ordem em baixa e alta frequência $(10-500 \mathrm{~Hz})$ no sinal EMG nos mesmos intervalos de tempo.

Todos os sinais (CVM e EMG) foram amostrados em $2000 \mathrm{~Hz}$. Analogamente à TDF, a TAE foi obtida através da curva EMG/tempo ( $\triangle E M G / \triangle$ tempo) nos intervalos 0-40, 0-60 e 0-80ms do início da iEMG.

A integração EMG (iEMG) foi iniciada 70ms antes do início da contração muscular para considerar o atraso eletromecânico. Os intervalos de tempo para cálculo da TAE foram reduzidos em relação à TDF devido a uma diminuição da amplitude EMG que comumente ocorre no início da contração muscular (80-100ms) ${ }^{18,13}$. A amplitude média EMG (EMG médio) foi obtida pela razão entre o iEMG e pelo tempo de integração (iEMG/tempo) nos intervalos 0-40, 0-60 e 0-80ms da integração do EMG. Todas as medidas foram mensuradas por um único avaliador.

\section{Análise estatística}

No programa estatístico SPSS versão 12.0 foram verificados a normalidade e homogeneidade dos dados com os testes de Shapiro-Wilke Levene, bem como calculadas as médias e desvios-padrão para todas as variáveis do estudo. No programa Excel 2.0 (Microsoft, Estados Unidos) foram calculadas: a TDF, o Impulso, a TAE e o EMG médio.

\section{RESULTADOS}

Os valores médios da força durante os primeiros 200ms de contração muscular em intervalos de 20ms apresentaram-se entre: 29,19 N.m no início da contração e 86,04 N.m nos 200ms da contração muscular, como pode ser observado na Figura 1.

Os valores da TDF ( $\Delta$ momento/ $\Delta$ tempo), avaliados durante $200 \mathrm{~ms}$, em intervalos de tempo de $20 \mathrm{~ms}$, apresentaram valores decrescentes na força de acordo com o tempo (Figura 2). Nos intervalos de 0-20 e 0-200 os valores observados distribuíram-se entre: 1459,42 à 430,21 N.m.s.-1.

A área sob a curva força-tempo, definida como o impulso contrátil, foi calculada no intervalo de 20 até $200 \mathrm{~ms}$ em intervalos de $20 \mathrm{~ms}$ desde o início da contração muscular (Figura 3), a delimitação da área de cada intervalo pode ser observada na Figura 1.
Nos intervalos de 0-40 a 0-200ms o impulso apresentou valores crescentes de 0,65 à 11,07 N.m.s, refletindo toda a história do tempo de contração muscular relacionada ao desenvolvimento da força.

ATAE ( $\triangle E M G / \Delta t e m p o$ ) foi obtida nos intervalos de 0-40, 0-60 e 0-80 ms relativos ao início da integração EMG e observou-se valores de 1320,88;

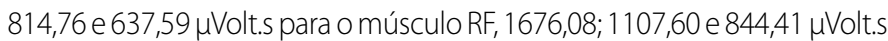
para oVL e 1747,63; 1224,19 e 914,09 $\mu$ Volt.s para o VM, conforme a Figura 4.

Ao se verificar a ativação neural eferente (iEMG/tempo de integração), a amplitude média EMG calculada nos intervalos de 0-40, 0-60 e 0-80ms no início da integração EMG (Figura 5) apresentou os valores de 24,93; 33,84 e 38,07 $\mu$ Volt para o RF, foram observados valores de 33,77, 44,76 e 50,32 $\mu$ Volt para o VL e observou-se valores de 37,07, 48,60 e 54,78 $\mu$ Volt para o VM. Assim, o músculo VM demonstrou maior ativação durante a extensão isométrica de joelho.

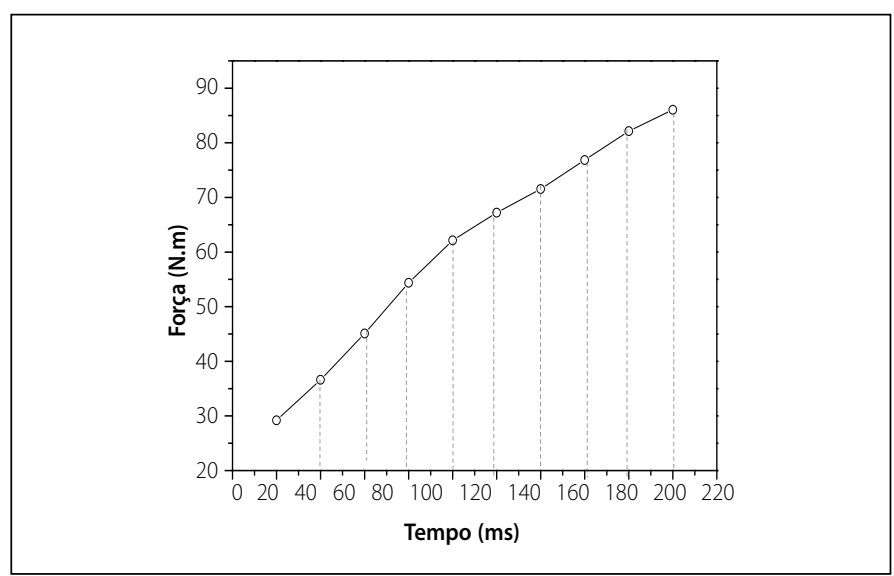

Figura 1. Curva força-tempo nos primeiros $200 \mathrm{~ms}$ da contração muscular ( $n=24)$.

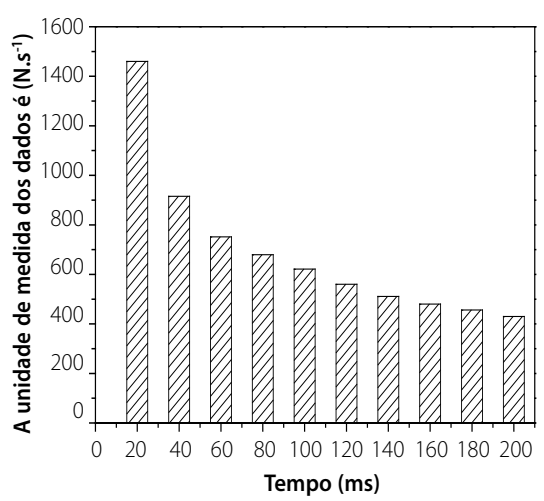

Figura 2. Taxa de desenvolvimento de força nos primeiros $200 \mathrm{~ms}$ da contração muscular $(n=24)$.

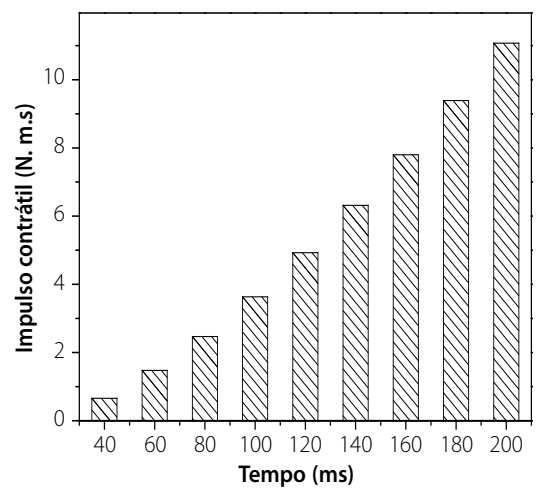

Figura 3. Impulso contrátil $(n=24) m$. 


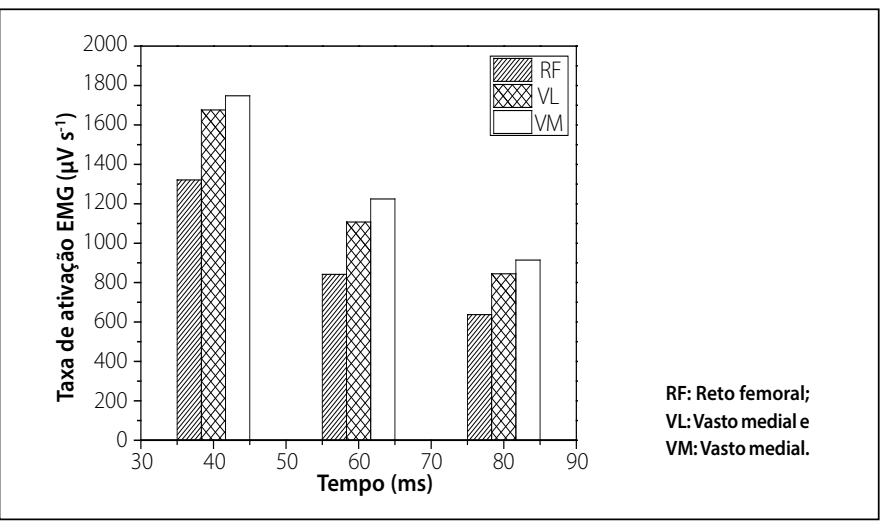

Figura 4. Taxa de ativação eletromiográfica ( $\triangle E M G / \Delta t e m p o)(n=24)$.

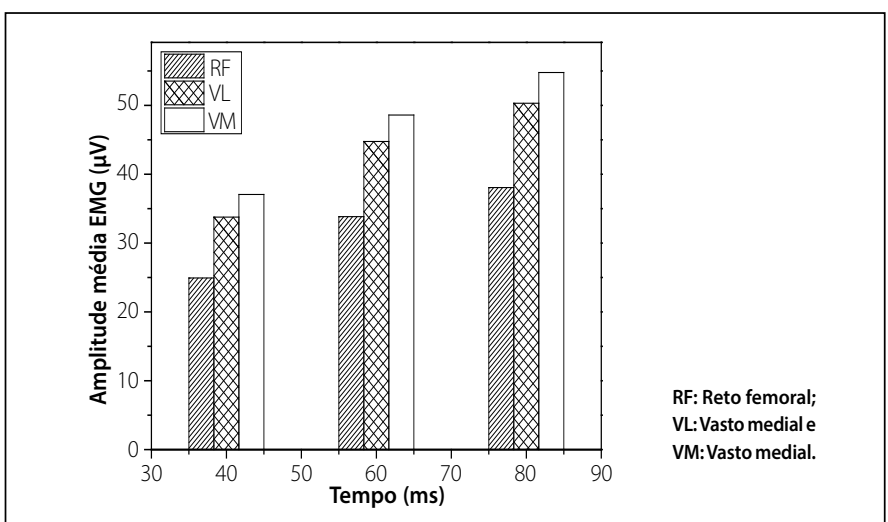

Figura 5. Amplitude média EMG $(n=24)$.

\section{DISCUSSÃO}

A proposta desse estudo foi verificar o comportamento da TDF e TAE em mulheres pós-menopausadas e fisicamente ativas. A curva força tempo, o impulso e a amplitude média EMG aumentaram com o tempo, enquanto observou-se valores decrescentes com o passar do tempo para a TDF e TAE para os músculos RF, VM e VL.

Em pessoas de meia idade e idosas em diversas situações da vida diária, como por exemplo: subir escadas, levantar da cadeira ou diante da necessidade de se reestabelecer a postura a fim de evitar quedas ${ }^{6}$ é desejável que a TDF se mantenha em níveis adequados, principalmente, durante a fase inicial (0-200ms) da contração muscular ${ }^{8}$, ao invés de apenas uma elevada força de contração isométrica. Quando comparados com sujeitos jovens, em idosos, a redução da força e da TDF pode chegar a 50 e 51\%, respectivamente, sendo ainda maior para as pessoas mais velhas ${ }^{19}$

Nesse estudo observou-se uma curva força/tempo crescente durante os primeiros 200ms da contração muscular (Figura 1), entretanto, quando calculada a TDF ( $\Delta$ momento/ $\Delta$ tempo) em intervalos de $20 \mathrm{~ms}$, pode-se observar um comportamento decrescente dos valores, com o decorrer do tempo (Figura 2). Embora a força seja elevada no momento muito inicial da contração muscular (0-20ms), a capacidade de gerar força rapidamente não é mantida, de modo que o aumento da força não se adapta a passagem do tempo. A redução da TDF observada neste estudo corrobora os verificados em outros estudos ${ }^{20-22}$. Suetta et al. ${ }^{23}$ investigou sujeitos idosos de ambos os sexos e observou comportamento semeIhante da TDF. Entretanto, em jovens, a TDF tende a apresentar valores crescentes com o aumento do tempo ${ }^{13,24,25}$.

A magnitude do impulso (Figura 3) reflete a velocidade angular e no presente estudo o impulso contrátil apresentou valores crescentes, refletindo assim, toda a história da contração a partir da integração de vários momentos da curva força/tempo, semelhante aos estudos de Suetta et al. ${ }^{23}$ em idosos e Aagaard et al. ${ }^{13}$ em jovens.
O processo de envelhecimento do sistema neuromuscular ao nível das unidades motoras pode explicar as possíveis causas dos diferentes comportamentos da TDF entre jovens e idosos. Com o passar dos anos, verifica-se uma atrofia seletiva dos NM das fibras tipo II ou de contração rápida ${ }^{18}$. Dessa forma, as fibras antes inervadas por NM com propriedades anaeróbias, sofrem um processo de remodelagem da unidade motora, e passam a ser inervadas por NM com propriedades oxidativas, alterando as propriedades das fibras tipo II para fibras tipo $\mathrm{I}^{26}$, o que pode influenciar diretamente a velocidade da geração de força no início da contração muscular.

De maneira semelhante à TDF, no decorrer da contração, a TAE ( $\triangle \mathrm{EMG} / \Delta$ tempo) apresentou valores decrescentes, nos músculos observados (VL, RF e VM) nos primeiros $80 \mathrm{~ms}$ do início da integração EMG (Figura 4). Aagaard et al. ${ }^{13}$ observou um comportamento crescente da TAE, entretanto, os autores utilizaram uma amostra de atletas jovens do sexo masculino submetidos ao treinamento de força. Lamentavelmente, nas buscas realizadas durante a revisão de literatura, não foram encontrados estudos que descrevessem os valores da TAE por intervalos de tempo em idosos.

A redução da TAE, durante os primeiros $80 \mathrm{~ms}$ da contração muscular, pode ser resultado de modificações na variabilidade de descarga das $\mathrm{UMs}^{8}$, ocasionada pela predominância de UMs lentas. Assim, de modo semelhante à TDF, a TAE não conseguiu acompanhar a passagem do tempo, demonstrando uma lenta ativação das fibras musculares, o que pode refletir em maior risco de queda, fraturas e dependência funcional especialmente em mulheres após a menopausa.

Quando calculado o EMG médio com a integração da área sob a curva $\triangle E M G / \triangle$ tempo, analogamente ao impulso contrátil, este demonstra o resumo de todo o período da contração muscular (0-80ms), pode-se observar valores crescentes no presente estudo para os músculos VM, RF e VL (Figura 5) semelhante aos estudos com jovens ${ }^{13}$ e contrariamente aos valores decrescentes observados em idosos do grupo controle do estudo de Gurjão et al. ${ }^{27}$, provavelmente pela característica da amostra do presente estudo ser fisicamente ativa no lazer. Não foram observados outros estudos que tenham examinado o EMG médio dos músculos VL, RF e VM em mulheres de meia idade ou idosas.

Interessantemente, mesmo apresentando um comportamento decrescente da TAE devido a não sustentação da velocidade de ativação durante os primeiros 200ms da contração muscular, as UMs parecem aumentar a taxa de descarga durante o intervalo de 0-80ms, ao se observar um comportamento crescente do EMG médio. Visto que o número de UM é reduzido em idosos ${ }^{28}$, durante as contrações voluntárias, a taxa de descarga poderia aumentar, mesmo que insuficientemente, para aperfeiçoar as propriedades de somação do músculo esquelético e assim, promover uma adaptação neurofisiológica para a perda de $\mathrm{UMs}^{18}$ que resulta no aumento do impulso contrátil.

O objetivo e métodos propostos para o presente estudo, não permitiram a verificação da capacidade adaptativa do sistema neuromuscular em relação a programas de intervenção, nem a comparação com mulheres jovens. Entretanto, verificaram-se resultados interessantes e pouco explorados, como por exemplo, o comportamento da variação da TDF, o impulso contrátil, a TAE e o EMG médio em mulheres de meia idade e idosas, após o período da menopausa, em músculos do membro inferior, devido à importância desses, no desempenho funcional e prevenção de quedas 20,22,29.

\section{CONCLUSÃO}

Verificou-se que em idosos, a taxa de desenvolvimento da força, apresenta um comportamento decrescente indicando a incapacidade do sistema neuromuscular dos músculos do quadríceps em gerar e man- 
ter o aumento rápido da força de modo a superar a passagem do tempo no período muito inicial da contração muscular. Da mesma forma, a TAE demonstrou-se decrescente nos primeiros 80ms da integração EMG, indicando um aumento insuficiente na ativação neural no início da contração. O EMG médio apresenta comportamento crescente resultando no aumento do impulso contrátil, entretanto, conservando ainda, características lentas, o que em pessoas de meia idade e idosas resulta em maior risco de quedas e dependência funcional.

\section{AGRADECIMENTOS}

Os autores agradecem ao Dr. Per Aagaard pelos esclarecimentos sobre o tema desse estudo, bem como ao apoio financeiro da Coordenação de Aperfeiçoamento de Pessoal de Ensino Superior - CAPES.

Todos os autores declararam não haver qualquer potencial conflito de interesses referente a este artigo.

CONTRIBUIÇÕES DOS AUTORES: Cada autor contribuiu individual e significativamente para o desenvolvimento do manuscrito. PMGP (0000-0002-7278-2385)* e AARG (0000-0002-4222-2264)* foram os principais contribuintes na redação do manuscrito, avaliaram os dados e discutiram os resultados. ALFA (0000-00026479-7851)* participou da concepção da proposta do estudo, coleta, tabulação e discussão dos resultados, ERLO (0000-0001-9310-2302)* e MGDSC (0000-00025924-6625)* realizaram a pesquisa bibliográfica, coleta, tabulação e revisão crítica da redação, MSCS (0000-0001-5566-3248)* participou ativamente da discussão dos resultados, revisou e aprovou a versão final do trabalho. *ORCID (Open Researcher and Contributor ID).

\section{REFERÊNCIAS}

1. Caserotti P. Strength training in older adults: changes in mechanical muscle function and functional performance. Open Sports Sci J. 2010;3:62-6.

2. Manini TM, Hong SL, Clark BC. Aging and muscle: a neuron's perspective. Curr Opin Clin Nutr Metab Care. 2013;16(1):21-6.

3. Van Kan GA, Chumlea CWM, Gillette-Guyonet S, Houles M, Dupuy CMSPT, Rolland Y. Clinical trials on sarcopenia: methodological issues regarding phase 3 trials. Clin Geriatr Med. 2011;17(3):471-82.

4. Janssen I. The epidemiology of sarcopenia. Clin Geriatr Med. 2011;27(3):355-63.

5. Holsgaard-Larsen A, Caserotti P, Puggaard L, Aagaard P. Stair-ascent performance in elderly women: effect of explosive strength training. J Aging Phys Act. 2011;19(2):117-36.

6. Aagaard P, Suetta C, Caserotti P, Magnusson SP, Kjaer M. Role of the nervous system in sarcopenia and muscle atrophy with aging: strength training as a countermeasure. Scand J Med Sci Sports. 2010;20(1):49-64.

7. Manini TM, Clark BC. Dynapenia and aging: an update. J Gerontol A Biol Sci Med Sci. 2012;67(1):28-40.

8. Klass M, Baudry S, Duchateau J. Age-related decline in rate of torque development is accompanied by lower maximal motor unit discharge frequency during fast contractions. J Appl Physiol. 2008;104(3):739-46.

9. Caserotti P, Aagaard P, Larsen JB, Puggaard L. Explosive heavy-resistance training in old and very old adults: changes in rapid muscle force, strength and power. Scand J Med Sci Sports. 2008;18(6):773-82.

10. Aagaard P. Training-induced changes in neural function. Exerc Sport Sci Rev. 2003;31:61-7.

11. Sirola J, Kröger H. Similarities in acquired factors related to postmenopausal osteoporosis and sarcopenia. J Osteoporos. 2011;2011:536735.

12. Aagaard P, Simonsen EB, Trolle M, Bangsbo J, Klausen K. Moment and power generation during maximal knee extensions performed at low and high speeds. Eur J Appl Physiol Occup Physiol. 1994;69:376-81.

13. Aagaard P, Simonsen EB, Andersen JL, Magnusson P, Dyhre-Poulsen P. Increased rate of force development and neural drive of human skeletal muscle following resistance training. J Appl Physiol. 2002;93(4):1318-26.

14. Aagaard P, Simonsen EB, Trolle M, Bangsbo J, Klausen K. Isokinetic hamstring/quadriceps strength ratio: influence from joint angular velocity, gravity correction and contraction mode. Acta Physiol Scand. 1995;154:421-7.

15. Winter DA. Biomechanics of human movement. John Willey \& Sons, Inc;; New York:1979.

16. Sahaly R, Vandewalle H, Driss T, Monod H. Surface electromyograms of agonist and antagonist muscles during force development of maximal isometric exercises-effects of instruction. Eur J Appl Physiol. 2003;89(1):79-84

17. Hermens HJ, Freriks B, Disselhorst-Klug C, Rau G. Development of recommendations for SEMG sensors and sensor placement procedures. J Electromyogr Kinesiol. 2000;10:361-74.

18. Roos MR, Rice CL, Vandervoort A.A. Age-related changes in motor unit function. Muscle Nerve. 1997;20:679-90.

19. Barry BK, Warman GE, Carson RG. Age-related differences in rapid muscle activation after rate of force development training of the elbow flexors. Exp Brain Res. 2005;162(1):122-32.

20. Gurjão ALD, Gobbi LTB, Carneiro NH, Gonçalves R, de Moura RF, Cyrino ES, et al. Effect of strength training on rate of force development in older women. Res Quartely Exerc Sport. 2012;83(2):268-75.

21. Amaral JF, Castro EA, Doimo LA, da Silva MV, Novo Jr JM. Mudanças no desenvolvimento temporal da força em mulheres de diferentes faixas etárias. Rev Bras Med Esporte. 2015;21(1):70-4.

22. Amaral JF, Castro EA, Mancini M, Doimo LA, Novo Jr JM. Taxa de desenvolvimento da força muscular de membros superiores e inferiores em mulheres idosas. Motricidade. 2012;8(\$2):454-61.

23. Suetta C, Aagaard P, Rosted A, Jakobsen AK, Duus B, Kjaer M, et al. Training-induced changes in muscle CSA, muscle strength, EMG, and rate of force development in elderly subjects after long-term unilatera disuse. J Appl Physiol. 2004;97(5):1954-61.

24. Farup J, Sørensen H, Kiølhede T. Similar changes in muscle fiber phenotype with differentiated consequences for rate of force development: endurance versus resistance training. Hum Mov Sci. 2014;34(1):109-19

25. Holtermann A, Roeleveld K, Vereijken B, Ettema G. The effect of rate of force development on maxima force production: acute and training-related aspects. Eur J Appl Physiol. 2007;99(6):605-13.

26. Akataki K, Mita K, Watakabe M, Ito K. Age-related change in motor unit activation strategy in force production: AA mechanomyographic investigation. Muscle Nerve. 2002;25(4):505-12.

27. Gurjão ALD, Gonçalves R, de Moura RF, Gobbi S. Acute effect of static stretching on rate of force development and maximal voluntary contraction in older women. J Strength Cond Res. 2009;23(7):2149-54

28. Kaya RD, Nakazawa M, Hoffman RL, Clark BC. Interrelationship between muscle strength, motor units, and aging. Exp Gerontol. 2013;48(9):920-5

29. Vandervoort AA. Aging of the human neuromuscular system. Muscle Nerve. 2002;25:17-25. 